\title{
A Comparison Between Rheological Properties of Intra-articular Hyaluronic Acid Preparations and Reported Human Synovial Fluid
}

\author{
Mat Nicholls · Ajay Manjoo - Peter Shaw · Faizan Niazi · \\ Jeffrey Rosen
}

Received: January 18, 2018 / Published online: March 14, 2018

(C) The Author(s) 2018

\section{ABSTRACT}

Introduction: This study aims to compare the properties of currently available intra-articular hyaluronate (IA-HA) products widely available in the USA to those of healthy knee synovial fluid with respect to their bulk rheological properties. We hypothesize that products would have differing rheological properties, with some more closely resembling the properties and physiological aspects of healthy joint fluid HA. Methods: We obtained reported HA product molecular weights, as well as measurements of

Enhanced content To view enhanced content for this article go to https://doi.org/10.6084/m9.figshare. 5943172.

M. Nicholls ( $\square)$

Virginia Mason Orthopedics and Sports Medicine, Seattle, WA, USA

e-mail: matn@mindspring.com

\section{A. Manjoo}

Division of Orthopaedics, McMaster University, Hamilton, ON, Canada

P. Shaw · F. Niazi

Ferring Pharmaceuticals Inc., Parsippany, NJ, USA

J. Rosen

Department of Orthopaedics and Rehabilitation,

New York Presbyterian Queens, New York, USA

J. Rosen

Department of Orthopaedic Surgery and

Rehabilitation, Weill Medical College of Cornell

University, New York, USA the presence of cross-linking, zero shear rate viscosity, shear thinning ratio, and crossover frequency for the following IA-HA products available in the USA: Euflexxa ${ }^{\circledR}$, Orthovisc ${ }^{\circledR}$, Supartz $^{\circledR}$, Monovisc $^{\circledR}$, Synvisc $^{\circledR}$, Synvisc-One $^{\circledR}$, Gel-One ${ }^{\circledR}$, and Hyalgan ${ }^{\circledR}$.

Results: Differences were seen between the study products across all of the investigated parameters. Hyalgan, Supartz, Orthovisc, and Euflexxa had a linear chain structure, while Synvisc, Synvisc-One, and Monovisc were crosslinked in structure. Molecular weight, shear rates, and crossover frequencies ranged widely across tested products, with values ranging from below to above those reported for healthy knee synovial fluid HA. When compared to healthy knee parameter values reported within the current literature, observed parameters for Euflexxa and Orthovisc were typically seen to be the most similar to healthy knee synovial fluid. When comparing Euflexxa and Orthovisc directly, Euflexxa was more often similar to the properties of healthy knee synovial fluid with respect to the observed parameters of molecular structure, shear rates, and crossover frequency. Conclusion: Available IA-HA products vary with respect to molecular weight, presence of cross-linking, shear rate dependency of viscosity, and crossover frequency. Since IA-HA treatment for osteoarthritis aims to restore synovial fluid back to original HA property characteristics, using HA supplements resembling healthy synovial fluid is a logical 
approach. Our findings demonstrate that Euflexxa is the most similar to healthy synovial fluid with respect to molecular structure, shear rates, and crossover frequency.

Funding: Ferring Pharmaceuticals, Inc.

Keywords: Hyaluronic acid; Knee; Osteoarthritis; Rheological properties; Synovial fluid; Viscosupplementation

\section{INTRODUCTION}

Knee osteoarthritis (OA) is a common cause of disability within developed countries [1]. The prevalence of knee OA in the USA is estimated to be $19.2 \%$ in individuals over the age of 45 and $43.7 \%$ in people over 80 years of age [1]. This disease creates significant direct and indirect burden on both the patient, as well as the cost to the healthcare system [2]. Knee OA can impact an individual's ability to participate in daily activities and can also result in a loss of ability to work [2]. Appropriate management of knee OA progression is important to allow for patients to continue with their daily routines without the implications of pain and functional limitations [2]. In order to appropriately manage knee OA, a thorough understanding of its causal mechanisms is required [3].

Radiographic findings in osteoarthritis of the knee are characterized by a reduction in joint space, as well as changes in the bone such as subchondral sclerosis and osteophyte (bone spur) formation. These changes are often associated with degradation of the cartilage within the knee joint, which can lead to pain and functional disability [4]. Previous research has found that hyaluronic acid (HA), a glycosaminoglycan polymer chain found within the synovial fluid, has shorter chain lengths, decreased molecular weight distribution, and lower concentration within osteoarthritic knees when compared to healthy knees $[3,5,6]$. The changes that arise from knee OA may also affect the rheological properties of the synovial fluid as a result of disease progression; these changes in rheological properties may also contribute to the progression of the disease [6]. The decrease in synovial HA molecular weight and concentration within the osteoarthritic knee is the primary disease component that the use of intra-articular hyaluronate (IA-HA) injection aims to address [7]. IA-HA is typically indicated and shown to be most effective in patients with mild to moderate knee OA. The rationale behind this treatment is that introducing new and concentrated HA into the synovial fluid replenishes the decreased HA concentration and average molecular weight present [7]. Clinical and preclinical research has indicated that HA injections provide therapeutic relief through chondral and subchondral protection, stimulating glycosaminoglycan production as well as anti-inflammatory, nociceptive, and jointcushioning effects [3].

Despite the various mechanisms in which IAHA provides therapy to the osteoarthritic knee, clinical studies report inconsistent outcomes and have resulted in conflicting clinical guideline recommendations [8]. There are differences in intrinsic properties between specific IA-HA products available on the market [9]. For example, current products range in molecular weight from 500 to $6000 \mathrm{kDa}$, and also vary in production method and presence of molecular cross-linking [9]. Some investigations have determined that products with a molecular weight greater than $3000 \mathrm{kDa}$ demonstrated significantly fewer discontinuations due to treatment-related adverse events than did hyaluronic acid products with a molecular weight less than $1500 \mathrm{kDa}$ [9]. Despite these intrinsic property differences between brands, all IA-HA products have been evaluated as equivalent in the development of clinical guidelines [9].

The current study aims to compare the properties of currently available IA-HA products widely available in the USA to those of healthy knee synovial fluid with respect to their bulk rheological properties. Since IA-HA aims to replenish the synovial fluid of osteoarthritic knees, IA-HA treatments that most closely resemble the molecular structure and viscoelastic properties of synovial fluid in healthy knees may be more appropriate in the management of knee OA. We hypothesize that tested products would have differing rheological properties, with some more closely resembling 
the properties and physiological aspects of healthy joint fluid HA.

\section{METHODS}

\section{Summary of Parameters Investigated}

We obtained reported HA product molecular weights, as well as measurements of presence of cross-linking, zero shear rate viscosity, shear thinning ratio, and crossover frequency for the following IA-HA products available in the USA: Euflexxa $^{\circledR}$ (Ferring Pharmaceuticals, USA), Orthovisc $^{\circledR}$ (DePuy Synthes, USA), Supartz ${ }^{\circledR}$ (Bioventus, USA), Monovisc ${ }^{\circledR}$ (DePuy Synthes, USA), Synvisc ${ }^{\circledR}$ (Sanofi, USA), Synvisc-One ${ }^{\circledR}$ (Sanofi, USA), Gel-One ${ }^{\circledR}$ (Zimmer, USA), and Hyalgan $^{\circledR}$ (Fidia Pharma, USA). The definition of each parameter is provided in Table 1 . This study does not contain any studies with human participants or animals performed by any of the authors.

All parameters were obtained with a TA AR 2000 EX Rheometer with cone-plate geometry (40 $\mathrm{mm}$ plate diameter and a $2^{\circ}$ cone angle) at room temperature, except molecular weight, which was determined from package inserts. The frequency sweeps were performed at strain amplitudes that were determined to be in the linear viscoelastic range. Only the viscosity data that reached equilibrium defined by the TA Rheometer algorithm were considered in the shear rate experiments. The IA-HA samples were kept at room temperature prior to use. The shear rate ranges explored were $0.001-1000 \mathrm{~s}^{-1}$ and the oscillatory frequency range was $0.001-100 \mathrm{~Hz}$, while the actual maximum and minimum ranges were determined by the ability of the rheometer to reach a stable reading. Each test run was duplicated with a fresh sample. Following each test the potential for evaporation or degradation to confound the results was tested by repeating a strain sweep at $1 \mathrm{~Hz}$. Results of the aforementioned analysis were compared to values for healthy knee synovial fluid reported within the current literature.

\section{RESULTS}

\section{Molecular Weight and Cross-linking}

Hyalgan, Supartz, Orthovisc, and Euflexxa have a linear chain structure. Of these, Euflexxa has a molecular weight of 2400-3600 kDa [10], Hyalgan has a molecular weight of $500-730 \mathrm{kDa}$ [11], Supartz has a molecular weight of

Table 1 Summary of analyzed parameters

\begin{tabular}{ll}
\hline Parameter & Definition \\
\hline $\begin{array}{l}\text { Molecular weight } \\
\text { Cross-linking }\end{array}$ & $\begin{array}{c}\text { The average mass of the HA molecules within the synovial fluid measured in kilodaltons } \\
\text { Cross-linking is the joining of molecules by covalent bonding, which causes alterations to physical } \\
\text { properties, such as viscosity and solubility }\end{array}$ \\
$\begin{array}{l}\text { Zero shear rate } \\
\text { viscosity }\end{array}$ & $\begin{array}{c}\text { The viscosity measured at a shear rate approaching zero. It represents the ability of an HA molecule to } \\
\text { resist permanent deformation under long-term loading }\end{array}$ \\
$\begin{array}{l}\text { Shear thinning } \\
\text { ratio }\end{array}$ & $\begin{array}{l}\text { The decrease in viscosity of the HA sample being tested when subject to a shear strain. This ratio } \\
\text { demonstrates how the viscosity of the sample is altered when under strains similar to those seen }\end{array}$ \\
Crossover & Crossover frequency observes changes in viscoelastic properties that the synovial fluid undergoes \\
frequency & during force changes in the knee. A common use is to demonstrate changes in the viscoelastic \\
& $\begin{array}{l}\text { properties of synovial fluid when increasing loading frequencies when converting from walking to } \\
\text { running }\end{array}$
\end{tabular}


620-1170 kDa [12], and Orthovisc has a molecular weight of 1000-2900 kDa [13] (Table 2). Synvisc, Synvisc-One, and Monovisc are cross-linked in structure. The average molecular weight of the soluble component (hylan A) of the entangled polymer Synvisc and Synvisc-One products was $6000 \mathrm{kDa}[14,15]$ and that of the monomers used in the manufacture of Monovisc was 1000-2900 kDa [16]. No molecular weight value was found for GelOne as a result of its highly cross-linked, gel-like structure [17].

\section{Shear Rate}

Euflexxa and Monovisc formulations had zero shear rate viscosities (at $0.1 \mathrm{~Pa}$ s) of 91.2 and 56.4 Pa s, respectively (Table 2). Hyalgan had a zero shear rate viscosity of $0.27 \mathrm{Pas}$, while Supartz had a zero shear rate viscosity of 3.07. The remaining products tested (Orthovisc, GelOne, Synvisc, and Synvisc-One) had a zero shear rate viscosity ranging from 120.8 to $191.7 \mathrm{~Pa}$ s. Synvisc and Synvisc-One demonstrated shear thinning ratios of 740.7 and 651.2, respectively. The shear thinning ratio of Euflexxa was 237.2, while Gel-One and Orthovisc's were 243.0 and 170.4 , respectively. The remaining three products [Hyalgan (2.33), Supartz (10.9), and Monovisc (51.5)] had lower shear thinning ratios than the aforementioned five products.

\section{Crossover Frequency}

The crossover frequencies of the analyzed products were Orthovisc, $0.16 \mathrm{~Hz}$; Euflexxa, $0.1 \mathrm{~Hz}$; Supartz, $3.98 \mathrm{~Hz}$; and Monovisc, $2.51 \mathrm{~Hz}$ (Table 2). Hyalgan's crossover frequency had to be extrapolated from the data and was greater than 10. Synvisc and Synvisc-One both had crossover frequencies of less than 0.01 after extrapolation from the analysis, while a crossover frequency was not observed for Gel-One within the oscillatory frequency tested within our investigation.

\section{DISCUSSION}

Healthy knee synovial fluid HA has a linear chain structure with an average HA molecular weight around $5000-6000 \mathrm{kDa}$, and primarily

Table 2 Rheological properties of tested HA products

\begin{tabular}{lllcll}
\hline Product & Mw (kDa) & $\begin{array}{l}\text { Cross- } \\
\text { linking }\end{array}$ & $\begin{array}{l}\text { Zero shear rate viscosity } \\
\left(\boldsymbol{\eta}_{\mathbf{0 . 1}}(\text { (Pa s) })\right.\end{array}$ & $\begin{array}{l}\text { Shear thinning ratio } \\
\left(\boldsymbol{\eta}_{\mathbf{0 . 1}} / \boldsymbol{\eta}_{\mathbf{2 5 0}}\right)\end{array}$ & $\begin{array}{l}\text { Crossover } \\
\text { frequency }(\mathbf{H z})\end{array}$ \\
\hline Hyalgan $^{\circledR}$ & $500-730$ & No & 0.27 & 2.33 & $>10^{\mathrm{e}}$ \\
Supartz $^{\circledR}$ & $620-1170$ & No & 3.07 & 10.9 & 3.98 \\
Monovisc $^{\circledR}$ & $1000-2900^{\mathrm{a}}$ & Yes & 56.4 & 51.5 & 2.51 \\
Orthovisc $^{\circledR}$ & $1000-2900$ & No & 120.8 & 170.4 & 0.16 \\
Euflexxa $^{\circledR}$ & $2400-3600$ & No & 91.2 & 237.2 & 0.10 \\
Gel-Onne $^{\circledR}$ & N/A & Yes & 190.2 & 243.0 & $\mathrm{~b}$ \\
Synvisc $^{\circledR}$ & $6000^{\mathrm{d}}$ & Yes & 191.7 & 740.7 & $<0.01^{\mathrm{e}}$ \\
Synvisc- $^{\mathrm{c}}$ & $6000^{\mathrm{d}}$ & Yes & 184.4 & 651.2 & $<0.01^{\mathrm{e}}$ \\
One $^{\circledR}$ & & & & & \\
\hline
\end{tabular}

${ }^{a}$ Molecular weight of the monomer used, not the cross-linked product

b Not observed

${ }^{c}$ Not reported as formulation is highly cross-linked

d Only reflective of the soluble portion

${ }^{\mathrm{e}}$ Extrapolated 
contributes to the viscoelastic properties of the synovial fluid $[7,18,19]$. Of the linear chain products, Euflexxa has the closest molecular weight to that of healthy knee synovial fluid (2400-3600 kDa), while the other linear chain products have lower molecular weights. Synvisc and Synvisc-One have similar molecular weights to healthy knee HA, however; the presence of cross-linking within Synvisc and Synvisc-One, as well as Monovisc and Gel-One, results in a branched structure of the HA chains. Only the soluble portion of these cross-linked products has been shown to be functional with respect to CD44 receptor interaction, making them dissimilar to the linear chain structure of healthy knee HA [20].

Healthy knee synovial fluid zero shear rate viscosity has been studied by several authors using samples aspirated from healthy knees and knees with OA. The observed zero shear rate viscosity for synovial fluid from a healthy joint ranges from 1 to $175 \mathrm{~Pa}$ s, while synovial fluid aspirated from $\mathrm{OA}$ joints ranges from 0.1 to $1 \mathrm{~Pa} \mathrm{~s}$ [21] as described by Fam et al. in the review article "Rheological properties of synovial fluids". A number of formulations (Hyalgan, Gel-One, Synvisc, Synvisc-One) had zero shear rate viscosities that fell outside of the range for healthy knee joints. Shear thinning ratios have been shown to range from 70 to 250 for synovial fluid aspirated from normal joints versus 5-40 for synovial fluid aspirated from joints with OA [21]. This investigation demonstrated that the shear thinning ratios of Euflexxa, Gel-One, and Orthovisc were the most similar to reported values for healthy knee synovial fluid, while Synvisc and Synvisc-One demonstrated shear thinning ratios much higher than that of healthy synovial fluid. These differences in cross-linked HA products from HA in healthy synovial fluid may result in a non-Newtonian fluid that does not operate similarly to HA within the knee environment.

The crossover frequency reported by Mazzucco et al. for healthy knee synovial fluid was $0.41 \mathrm{~Hz}$, while synovial fluid from OA knees had a crossover frequency of $1.8 \mathrm{~Hz}$ [22]. The physiological frequencies of the knee joint in the context of different physical activities have been described to be $0.5 \mathrm{~Hz}$ for walking and
$3 \mathrm{~Hz}$ for running [23]. The crossover frequencies of 0.16 and $0.1 \mathrm{~Hz}$ of Orthovisc and Euflexxa were the closest to the values of healthy knee synovial fluid. Supartz and Monovisc demonstrated much higher crossover frequencies than healthy knee synovial fluids. The extrapolated crossover frequencies of Synvisc and SynviscOne were much smaller than that of healthy knee synovial fluid, while Hyalgan's crossover frequency was much larger. This shows that the cross-linked products demonstrate a lack of viscous mode, and demonstrate only elastic properties in the normal operation function of the knee, which agrees with results in previous literature [23]. Conversely, Hyalgan does not demonstrate an elastic mode within the normal functional range of the knee, as it primarily demonstrates a viscous mode characteristic. Gel-One did not reach a crossover frequency within our testing, likely due to the gel-like structure of the product. In the context of physical activity, some of the above crossover frequencies are less than the physiological frequency for walking $(\approx 0.4 \mathrm{~Hz})$ and exceed those for running $(\approx 5 \mathrm{~Hz})$.

This analysis suggests that Euflexxa and Orthovisc are most similar to healthy knee HA in terms of the viscoelastic parameters studied. When comparing these two products, Euflexxa has a higher average molecular weight than Orthovisc and closer to the molecular weight of healthy knee synovial fluid. Other non-crosslinked products have lower molecular weight, viscosity, and higher crossover frequency results than typically seen within healthy knee OA. Cross-linking creates an HA molecule tertiary structure that is not representative of the HA structure or qualities typically seen within healthy knee synovial fluid. Cross-linked weak gel-type products typically did not demonstrate a zero shear rate viscosity within the range of shear rates studied, had a higher shear thinning ratio, and had a lower crossover frequency than healthy knee synovial fluid. If the goal of IA-HA treatment is to relieve pain and OA symptoms through replenishing and increasing the HA molecular weight distribution and concentration in the synovial fluid HA of osteoarthritic knees, a product that closely resembles healthy knee HA may be the most appropriate choice. 
The reason for this is that the knee operates within a certain range of pressures and frequencies. Since IA-HA products are trying to match the parameters of the native lubricant, the ideal product should resemble as close as possible the native zero shear viscosity, shear thinning, and crossover frequency.

This study is limited by its use of duplicate samples, which was done instead of triplicate as a result of available material. Duplicate testing was done using samples from two different syringes to help address this issue and provide additional robustness to the analysis; however, these two syringes were from the same manufacturing lot for each product. This study is limited by the lack of correlation to the clinical impacts of these results. It is inferred that IA-HA treatments that most closely resemble healthy knee synovial fluid HA may be the most appropriate products; however, this is not clear with respect to evidence of clinical outcomes. Additionally, the use of previously obtained values of healthy and OA knee values from the literature is not ideal, as there may be potential differences in the methodology of the investigations when compared to the methodology used in this study. We included comparisons to studies that reported similar methodology to decrease the impact of this limitation; however, we cannot be certain that the study conditions were equal, and as these methodologies were taken from other publications, we did not include them in our results table (Table 2). Ideally, samples of healthy human synovial fluid would have been tested; however, this was not feasible at the time of analysis for this study. A limitation to consider is the temperature in which these tests were conducted. These properties were tested at room temperature; however, the rheological properties may be affected by the temperature within the joint. Despite this limitation, we believe that testing all products under the same conditions still provides valuable information regarding their properties, despite it not being at body temperature. Moreover, the studies that had healthy knee HA data used similar analysis methods under similar conditions and temperatures, making the results more comparable. The effects of molecular weight, cross-linking, shear rate, and crossover frequency require further investigation with respect to clinical implications under physiological friction forces within the knee joint. It is also of importance to investigate the effect that these rheological property results have on mechanisms in which HA provides relief, such as chondral and subchondral protection, anti-inflammatory, nociceptive, glycosaminoglycan production, and joint-cushioning effects [3]. This study helps demonstrate which products are most similar to healthy knee HA rheologically; however, there is a need for further work to understand how the differences in rheological properties correlate with actual treatment outcomes including pain relief, function, and effects on the cartilage itself.

\section{CONCLUSIONS}

Available HA products vary with respect to molecular weight, presence of cross-linking, shear rate dependency, and crossover frequency. Euflexxa and Orthovisc demonstrate the most similar results across these measures when compared to HA found within the healthy knee synovial fluid, yet Euflexxa's molecular weight is closer than Orthovisc's to that of the molecular weight of healthy knee HA. Since IAHA treatment for osteoarthritis aims to restore synovial fluid back to original HA property characteristics, the use of a product that most closely resembles healthy knee HA is a logical choice.

\section{ACKNOWLEDGEMENTS}

Funding. This study was funded by Ferring Pharmaceuticals Inc. Article processing charges were funded by Ferring Pharmaceuticals Inc. All authors had full access to all of the data in this study and take complete responsibility for the integrity of the data and accuracy of the data analysis.

Authorship. All named authors meet the International Committee of Medical Journal 
Editors (ICMJE) criteria for authorship for this article, take responsibility for the integrity of the work as a whole, and have given their approval for this version to be published.

Medical Writing and/or Editorial Assistance. The authors would like to thank Mark Phillips of Global Research Solutions Inc. for his medical writing assistance that was funded by Ferring Pharmaceuticals Inc. The coauthors would also like to thank Izi Bruker for his assistance with the analysis and review of earlier versions of the manuscript.

Disclosures. Mat Nicholls is a consultant for Ferring Pharmaceuticals, Inc. Jeffery Rosen is a speaker trainer for Ferring Pharmaceuticals, Inc. Peter Shaw is a paid employee of Ferring Phamaceuticals Inc. Faizan Niazi is a paid employee of Ferring Pharmaceuticals Inc. Ajay Manjoo has nothing to disclose.

Compliance with Ethics Guidelines. This study does not contain any studies with human participants or animals performed by any of the authors.

Open Access. This article is distributed under the terms of the Creative Commons Attribution-NonCommercial 4.0 International License (http://creativecommons.org/licenses/ by-nc/4.0/), which permits any noncommercial use, distribution, and reproduction in any medium, provided you give appropriate credit to the original author(s) and the source, provide a link to the Creative Commons license, and indicate if changes were made.

\section{REFERENCES}

1. Litwic A, Edwards MH, Dennison EM, Cooper C. Epidemiology and burden of osteoarthritis. Br Med Bull. 2013;105:185-99.

2. Hunter DJ, Schofield D, Callander E. The individual and socioeconomic impact of osteoarthritis. Nat Rev Rheumatol. 2014;10:437-41.

3. Altman RD, Manjoo A, Fierlinger A, Niazi F, Nicholls $M$. The mechanism of action for hyaluronic acid treatment in the osteoarthritic knee: a systematic review. BMC Musculoskelet Disord. $2015 ; 16: 321$.

4. Cicuttini FM, Wluka AE, Stuckey SL. Tibial and femoral cartilage changes in knee osteoarthritis. Ann Rheum Dis. 2001;60:977-80.

5. Balazs E. The physical properties of synovial fluid and the special role of hyaluronic acid. 2nd ed. Philadelphia: JB Lippincott; 1982.

6. Kosinska MK, Ludwig TE, Liebisch G, et al. Articular joint lubricants during osteoarthritis and rheumatoid arthritis display altered levels and molecular species. PLoS ONE. 2015;10:e0125192.

7. Watterson JR, Esdaile JM. Viscosupplementation: therapeutic mechanisms and clinical potential in osteoarthritis of the knee. J Am Acad Orthop Surg. 2000;8:277-84.

8. Altman RD, Schemitsch E, Bedi A. Assessment of clinical practice guideline methodology for the treatment of knee osteoarthritis with intra-articular hyaluronic acid. Semin Arthritis Rheum. 2015;45:132-9.

9. Altman RD, Bedi A, Karlsson J, Sancheti P, Schemitsch E (2016) Product differences in intra-articular hyaluronic acids for osteoarthritis of the knee. Am J Sports Med 2016;44(8):2158-65.

10. Euflexxa Package Insert: Product Code 55566-41001.

11. Hyalgan Sodium Hyaluronate Package Insert: Product Code 684073/3.

12. Supartz (sodium hyaluronate) Package Insert: Product Code 6CH40403.

13. Orthovisc High Molecular Weight Hyaluronan Package Insert: Product Code 500-254/A.

14. Synvisc-One Hylan G-F20 Package Insert: Product Code 70240104.

15. Synvisc Hylan G-F20 Package Insert: Product Code 70230607.

16. Monovisc High Molecular Weight Hyaluronan Package Insert: Product Code 500-299/A.

17. Gel-One Cross Linked Hyaluronate Package Insert: Product Code 6LA402000.

18. Fessler JH, Fessler LI. Electron microscopic visualization of the polysaccharide hyaluronic acid. Proc Natl Acad Sci USA. 1966;56:141-7. 
19. Dahl LB, Dahl IM, Engstrom-Laurent A, Granath K. Concentration and molecular weight of sodium hyaluronate in synovial fluid from patients with rheumatoid arthritis and other arthropathies. Ann Rheum Dis. 1985;44:817-22.

20. Jackson DW, Simon TM. Intra-articular distribution and residence time of Hylan A and B: a study in the goat knee. Osteoarthr Cartil. 2006;14:1248-57.

21. Fam H, Bryant JT, Kontopoulou M. Rheological properties of synovial fluids. Biorheology. 2007;44:59-74.
22. Mazzucco D, McKinley G, Scott RD, Spector M. Rheology of joint fluid in total knee arthroplasty patients. J Orthop Res. 2002;20:1157-63.

23. Finelli I, Chiessi E, Galesso D, Renier D, Paradossi G. A new viscosupplement based on partially hydrophobic hyaluronic acid: a comparative study. Biorheology. 2011;48:263-75. 